\title{
Anfang und Umbau des Gewässernetzes zwischen Wupper und Sieg (Oberbergisches Land, rechtsrheinisches Schiefergebirge)
}

\author{
HeLlmut GRABERT*)
}

\begin{abstract}
Kurzfassung: Die Herausbildung des rezenten Gewässernetzes im Rheinischen Gebirge setzte nach der Hebung der Rheinischen Masse im Gefolge der alpinen Orogenese ein, ist also spätoligozänen Alters. Noch im Alttertiär erfolgte die Entwässerung des tiefgreifend zersetzten Variszikums über weitgespannte Spülflächen, in denen sich die Drainageströme mäandrierend bewegten. Mit der Hebung wurden die Flüsse in geführten Mäandern tiefergelegt und zum Rhein hin ausgerichtet. Dieser Umschlag von der Spülflächenbildung zur linearen Flußerosion wird als „Morphogenetischer Umbruch" bezeichnet und ab dem Ende des Oligozäns datiert. Der starke Miozän-Vulkanismus steht mit der Hebung der Rheinischen Masse in ursächlicher Beziehung.
\end{abstract}

\section{[Beginning and development of the stream network situated between the rivers Wupper and Sieg in the southern part of the Rhenish massif right to the river Rhine]}

\begin{abstract}
The development of the recent drainage system in the Rhenish Mountains started during Late Oligocene by the Alpine orogenesis. Still the Early Tertiary time the drainage on the strongly weathered Variscans took place by unlarged flushing areas and meandring rivers. During the uplift of the Rhenish Massif these rivers in its meandring manner were leaded down to the Variscan body, and directed toward the Rhine main river. The change from the system of flushing areas to linear erosion by river drainage is called the "Morphogenetic Upheaval", dated Late Oligocene. The volcanism during the Miocene was caused by the uplift of the Rhenish Massif.
\end{abstract}

\section{Einleitung}

Der Anfang und der Umbau des rechtsrheinischen Gewässernetzes ist einerseits nur im Zusammenhang mit der Entwicklung des Rheintales - zwischen Bingen und Bonn - zu entschlüsseln, andererseits werden dafür aber auch stratigraphische Fixpunkte benötigt, um den zeitlichen Ablauf aufzeigen zu können.

Die Verebnungs- und Terrassen-Abfolge des Rheintales ist seit knapp hundert Jahren (PHILIPPson 1899)

*) Anschrift des Verfassers: Prof. Dr. H. Grabert, Haselbuschweg 5, 47804 Krefeld bekannt und in vielen Arbeiten differenziert worden. Auch für die rechtsrheinischen Nebenflüsse, insbesondere der Sieg (KNUTH 1922, SCHRÖDER 1965, Gramsch 1978) und der Agger (Hoos 1936, SchröDER 1969, FEY 1974), sowie für das Bergische Land (NICKE 1981, 1983) liegen umfangreiche Bearbeitungen vor. Was bisher fehlt, ist ein verläßlicher Fixpunkt in der Zeitskala, auf den sich die Bildungen der Verebnungs- und Terrassenflächen beziehen lassen. Dieser Fixpunkt wird mit einem morphogenetischen Profil vom Hohen Westerwald bis zum Ebbe-Gebirge vorgelegt.

Bei der Betrachtung der Verebnungs- und Terrassenflächen fällt immer wieder der Unterschied beider Flächen auf: Verebnungsflächen tragen in der Regel keine Sedimente (vom späteren, äolisch aufgebrachten pleistozänen Löß abgesehen), weisen dafür einen starken Zersatz des Gebirges mit fossilen Bodenbildungen auf, sie werden in die Tertiärzeit mit ihrem „tropoiden", also feuchtwarmen bis feucht-gemäßigten Klima gestellt. Die Terrassenflächen hingegen, die sich eng an den heutigen Flußlauf halten, tragen Schotter und gehören dem Pleistozän, gelegentlich noch dem Holozän an.

Dieser Umschlag in der Ausbildung von Verebnungs- zu Terrassenflächen ist bedingt durch den Aufstieg der Rheinischen Masse aus einem tertiärzeitlich tiefgründig zersetzten Gebirgsrumpf in niederer Seehöhe zu einem Mittelgebirge mit starker Zertalung und Schotterbildung. Eingesetzt hat diese Hebung als „Plateau Uplift“ (Fuchs et al. 1983) in der mittleren Tertiärzeit, sie wird begleitet von einem heftigen Basalt-Vulkanismus im Miozän. Der Umschlag von den Verebnungs- zu den Terrassenflächen wird als „Morphogenetischer Umbruch“ bezeichnet und weitgehend dem mittleren Miozän zugeordnet (BIRKENHAUER 1970).

Keineswegs scheint nun die Hebung der rheinischen Masse kontinuierlich vonstatten gegangen zu sein. Allein der Basalt-Vulkanismus (im Miozän zwischen 30 und 18 Mio. Jahren) und der pleistozäner Nachläufer (von 1,0 bis 0,3 Mio. Jahren) lassen auf langdauernde, aber auch unterschiedliche Aufwärts- 


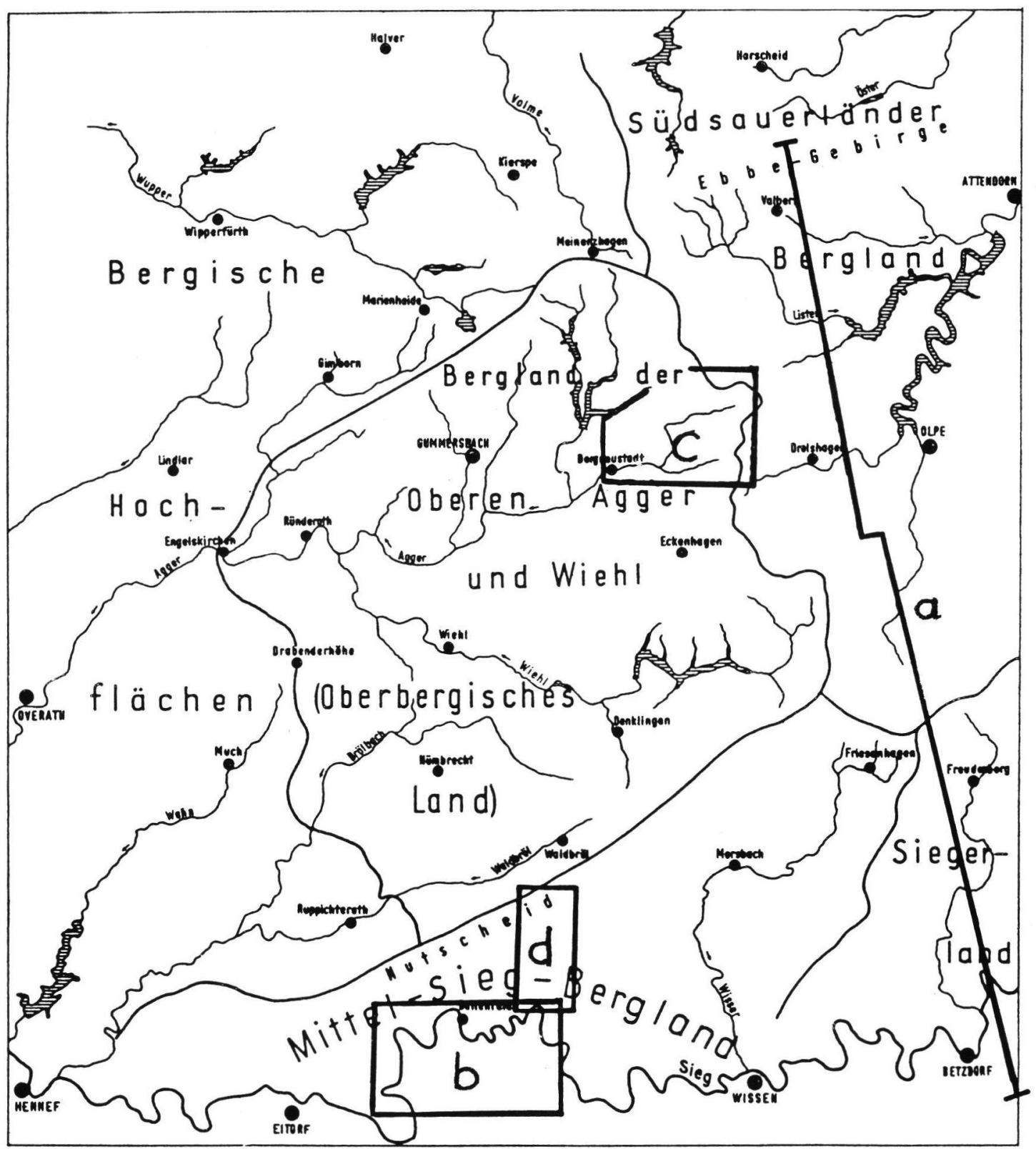

Abb. 1: Die naturräumliche Gliederung des Landes zwischen Wupper und Sieg

a: Das morphogenetische Profil „Hoher Westerwald - Ebbe-Gebirge“ (Abb. 2), b: Der Talmäanderbogen der Sieg bei Dattenfeld (Abb. 3); c: Die Pernzer Talung am Oberlauf der Agger bei Bergneustadt (Abb. 4); d: Die Spurkenbacher Talung des Westert-Baches (Abb. 5); +240 m (Hennef), + $460 \mathrm{~m}$ (Betzdorf), > $660 \mathrm{~m}$ (Ebbe): Höhenlage der präbasaltischen Verebnungsfläche

Fig. 1: The landscape of the region between the Wupper and the Sieg river. Drawing in the studied areas

a: The morphogenetic perfil „Hoher Westerwald - Ebbe Mountains“ (fig. 2); b: The valley arch of meanders in the Sieg river system near Dattenfeld (fig. 3); c: The Pernze head valley in the Agger-Dörspe drainage system (fig. 4); d: The erosion surface Spurkenbach and its capture by the Westert brook (fig. 5)

bewegungen schließen. Solche Ereignisse müssen sich auf das Entwässerungsnetz des aufsteigenden Gebirges auswirken, wobei auch plötzliche Laufveränderungen aufgetreten sind.

Beispiele aus geologisch junger Zeit werden hier vorgestellt: der Talmäanderbogen der Sieg zwischen Au und Eitorf, das Anzapfen der Spurkenbacher Talung am Nutscheid-Kamm und die bis in holozäne Zeit andauernde Laufveränderungen in der Pernzer Talung bei Bergneustadt an der Agger (Abb. 1). 


\section{Anfang und Umbau des Gewässernetzes}

Die Entwicklung und damit auch die Veränderung des Gewässernetzes im rechtsrheinischen Schiefergebirge (und natürlich auch im linksrheinischen, hier nicht behandelten) ist abhängig von der Entstehung des Rheintales. Der Rhein ist als Fluß keineswegs so alt und einheitlich, wie er sich derzeit darstellt. Im Gegenteil: dieser Strom ist ein Produkt der Eis- und Nacheiszeit und besitzt außerdem eine „unlogische" Entwässerungsrichtung. Aus den Alpen als Hochrhein kommend, müßte er eigentlich der Donau zufließen, die das Alpenvorland als natürliche Rinne zur Entwässerung nutzt und nach Osten abfließt. Der direkte Weg über die Rhône zum Mittelmeer ist heute verbaut, war aber einst im Alttertiär offen.

Der heutige Rhein ist aus drei Teilstücken zusammengesetzt: aus dem einst zur Donau hin orientierten Hochrhein, aus dem über den Rhône-Graben zum Mittelmeer hin ausgerichteten Oberrhein und aus dem zur Nordsee abfließenden Mosel-Mittelrhein-System (Quitzow et al. 1962).

Stark beeinflußt worden sind alle drei Teilstücke durch die klimatischen Veränderungen während und seit der Eiszeit, insbesondere auch durch deren pleistozäne Ablagerungen. Aber schon früher, in der mittleren Tertiärzeit, wirkten Kräfte auf die Gcstaltung der Landschaft ein, die so gegensätzlich waren, daß man den Übergang von dem einen in den anderen Klimabereich als „Morphogenetischen Umbruch" bezeichnete (BIrKenHauer 1970, GramsCH 1978). Dieser „Umbruch“ hat seine Ursache in der Alpen-Faltung, die sich im Mittelrhein-Gebiet als Hebung der rheinischen Masse verdeutlicht und mit dem (miozänen) Basalt-Vulkanismus im Westerwald, der Rhön und des Vogelsberges sowie der Westeifel in engem Zusammenhang steht.

Irgendwann nach diesem Morphogenetischen Umbruch entstand inmitten der langsam aufsteigenden Rheinischen Masse eine Geländedepression, welche sich in die älteren Gebirgsrumpfflächen einsenkte; sie zeigt zum ersten Male eine „rheinische“, also nach Norden gerichtete Ausformung. Auf diese, als Ur-Niederrhein zu bezeichnende Drainage richtet sich von nun an die Entwässerung des kommenden Rheinischen Schiefergebirges aus, und natürlich auch, vielleicht mit einer zeitlichen Verzögerung, die der tributären Zuflüsse. Zeitlich eingrenzen kann man diesen neu entstandenen Vorfluter durch die Ablagerungen der als „Pliozän“ aufgefaßten, weißen Quarzkiese, die sich am Rande des Westerwaldes erhalten haben; sie weisen nämlich noch keine Beziehungen zu den Rhein-Terrassen auf und scheinen nach Süden hin orientiert zu sein.

Auch die großen Nebenflüsse wie die Mosel, die Lahn, aber auch die Sieg, haben als erste Anlage sol- che Depressionen benutzt, wobei aber bis heute nicht geklärt ist, wodurch solche morphologischen Strukturen entstanden sein könnten. Unter ihnen tektonische Elemente zu vermuten, wurde für die Nord-Süd gerichtete Bergische Muldenzone diskutiert (Grabert 1983), in der die Agger, Sieg und Wupper fließen.

Bei der Betrachtung der Reliefgenese schälen sich zwei unterschiedlich ausgebildete Flächengruppen heraus:

1. ein älteres, flächenhaftes System ohne aufgebrachte klastische Sedimente; es wird als das Altflächenrelief bezeichnet; und

2. das jüngere, in dem eigentlichen Gebirgsrumpf eingetiefte, sich eng an den heutigen Flußlauf haltende Terrassensystem mit Schotterbedeckung; man bezeichnet dieses als Talnetz.

Im Rheinischen Schiefergebirge wird die ältere Gruppe als Trogtal bezeichnet (Quitzow et al. 1962) und sinngemäß gilt das auch für seine Nebenflüsse (z. B. für die Sieg [SCHRÖDER 1969]), die jüngere in das Hochtal, Plateautal und Engtal. Für eine befriedigende Terrassen-Chronologie wird eine markante Zeitmarke gesucht, die nun im südlichen Siegerland, wo mit dem miozänen Basalt des Hohen Westerwaldes eine sogar absolut datierbare Zeitmarke gegeben ist; an diese wird das Morphogenetische Profil „Hoher Westerwald - Ebbe-Gebirge" angehängt.

\section{Profil „Hoher Westerwald - Ebbe-Gebirge“ (Abb. 2)}

Dort, am Nordrande des Hohen Westerwaldes, liegt bei +460 m NN der „oligozäne Klebsand“, vermutlich aus der Chatt-Stufe, auf einem stark verwitterten devonischen Gesteinssockel; der auflagernde miozäne Basalt hat nach den Untersuchungen von Lippolt (1983: Abb. 2) ein Alter zwischen 30 und 18 Mio. Jahren, mit einem Maximum um 25 Mio. Jahre (die pleistozäne Nachläuferphase datiert zwischen 1,0 und 0,3 Mio. Jahren).

Diese jungoligozäne 460-m-Verebnung des Hohen Westerwaldes - z. B. im Gebiet der TK 25 Betzdorf 5213 - ist tiefgründig zersetzt und ist pedologisch durch Weißlehme und Bleichungszonen ausgezeichnet, sowie durch Rotlehm-Bildung infolge einer Eisenanreicherung in der Nähe des wasserstauenden devonischen Gebirgskörpers.

Nach Norden, im Gebiet der TK 25 Freudenberg 5113 , ist diese „oberoligozäne“ Fläche durch ihre exotische Bodenbildung aus Bleichungszonen und Rotlehmen noch deutlich erkennbar, steigt aber langsam auf $+480 \mathrm{~m} \mathrm{NN}$ (der Giebelwald mit +527 $m$ NN ragt als Härtling aus dieser Verebnungsfläche heraus) und läßt sich im nördlich anschließenden Gebiet der TK 25 Kreutal 5013 nicht mehr nachweisen. Bei Belmicke, mit $+464 \mathrm{~m} \mathrm{NN}$ an der Grenze zur 

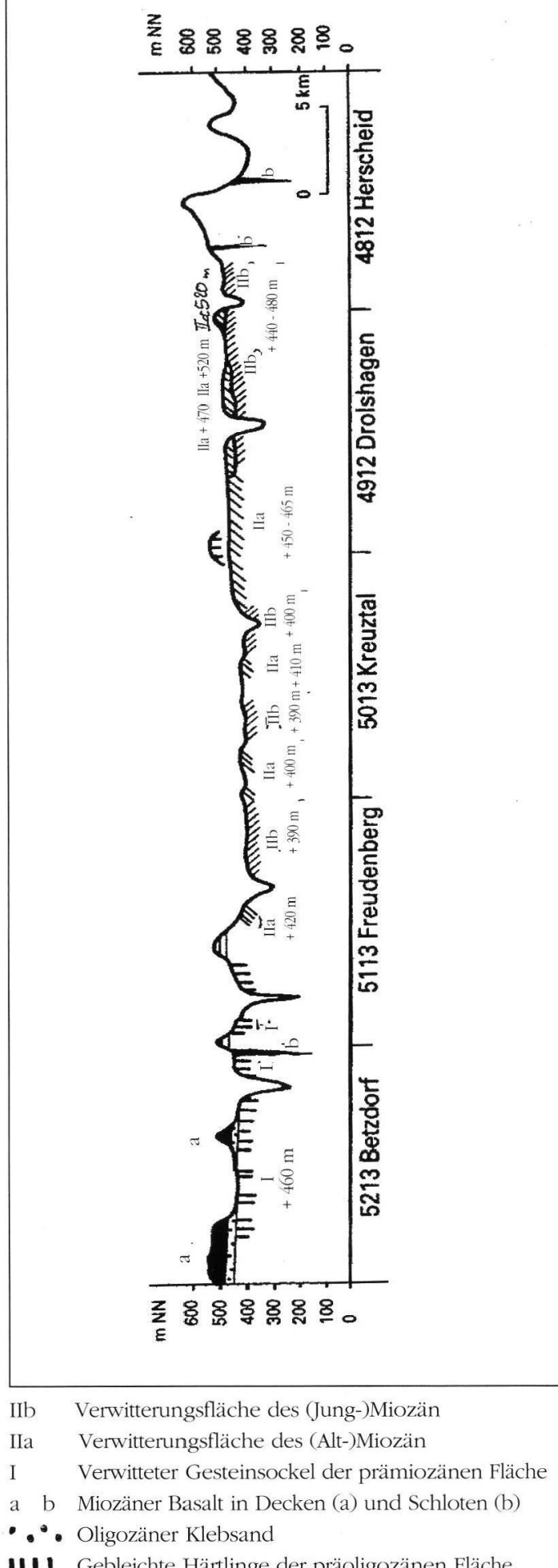

II 1 Gebleichte Härtlinge der präoligozänen Fläche

Abb. 2: Das morphogenetische Profil „Hoher Westerwald Ebbe-Gebirge"

Fig. 2: The morphogenetic perfil "Hoher Westerwald - Ebbe Mountains
TK 25 Drolshagen 4912 (Profil nach NE versetzt), ist das devonische Grundgebirge nur geringfügig und dann der heutigen Bodenbildung entsprechend rostbraun verwittert. Verlängert man das morphogenetische Profil nach Norden bis in das Ebbe-Gebirge hinein (TK 25 Herscheid 4812), sind auch dessen höchste Erhebungen, die Nordhelle mit $+663 \mathrm{~m} \mathrm{NN}$ und der Rehberg mit $+648 \mathrm{~m} \mathrm{NN}$, frei von jeglicher fossiler, also tertiärzeitlicher Bodenbildung: Bleichungszonen und Rotlehme fehlen.

Die einst vorhandenen Weißlehme liegen heute am hohen Hang auf sekundärer Stätte und haben zu umfangreichen Vernässungen mit Hochmooren geführt. Am morphogenetischen Profil (Abb. 2) wird deutlich, wie stark die Hebung der Rheinischen Masse ist. Liegt noch am Südrande des Siegerlandes die präbasaltische Auflagerungsfläche des miozänen Westerwald-Basaltes bei $+460 \mathrm{~m} \mathrm{NN}$, ist diese nach $50 \mathrm{~km}$ im Ebbe-Gebirge bei mehr als $+660 \mathrm{~m} \mathrm{NN}$ nicht mehr vorhanden, muß also höher gelegen haben. Da die (miozäne) IIa-Fläche im Gebiet von Freudenberg schon bei $+420 \mathrm{~m} \mathrm{NN}$, am Fuße des Ebbe-Gebirges schon bei ca. $+470 \mathrm{~m} \mathrm{NN}$ bis $+525 \mathrm{~m} \mathrm{NN}$ liegt, errechnet sich daraus ein Hebungsbetrag von mindestens $50 \mathrm{~m}$ (bis max. $105 \mathrm{~m}$ ?) auf einer Entfernung von ca. $50 \mathrm{~km}$.

Aber nicht nur in der Nordsüd-Erstreckung macht sich die Hebung der Rheinischen Masse bemerkbar, sondern auch in ostwestlicher. Bei Uckerath, unweit Hennef am Rande des Bergischen Landes zur Niederrheinischen Bucht, liegen oligozäne Kiese vermutlich von Chatt-Alter bei $+240 \mathrm{~m} \mathrm{NN}$. Bezieht man diese Auflagerungsfläche auf gleichalte Vorkommen des Hohen Westerwaldes - dort bei $+460 \mathrm{~m}$ NN gelegen -, erhält man einen Höhenunterschied von ca. $120 \mathrm{~m}$ auf eine Entfernung von ca. $50 \mathrm{~km}$.

Nun liegen diese oligozänen Kiese heute bei $+240 \mathrm{~m}$ $\mathrm{NN}$, sind aber in dieser Höhe nicht abgelagert worden, vielleicht nicht allzu hoch über dem damaligen Meeresspiegel. Die älteren marinen Rupel-Tone und Grafenberger Sande zwischen Köln und Düsseldorf liegen heute ca. $50 \mathrm{~km}$ flußabwärts bei ca. $+150 \mathrm{~m}$ Seehöhe; damals stand also dort das Meer. Am Ende des Oligozän setzte dann die eigentliche Hebung der Rheinischen Masse ein, verstärkte sich gegen Ende des Miozän und dauert bis heute noch an. Das Einsinken der Niederrheinischen Bucht - in der die Absenkung Tertiär-Basis bis weit unter Meeresspiegel-Niveau erfolgte - ist eine sekundäre Ausgleichbewegung (sie wird hier nicht näher betrachtet). Summiert man alle Beträge, beginnend an der Basis des dem verwitterten Festland aufgesetzten miozänen Basaltes, dann erhält man einen Hebungsbetrag für das zentrale Rheinische Schiefergebirge von ca. $300 \mathrm{~m}$ (und mehr), ein Betrag, der mit allen bisherigen Kenntnissen (zusammengefaßt bei FucHs et al. 1983: Plateau Uplift) übereinstimmt. 


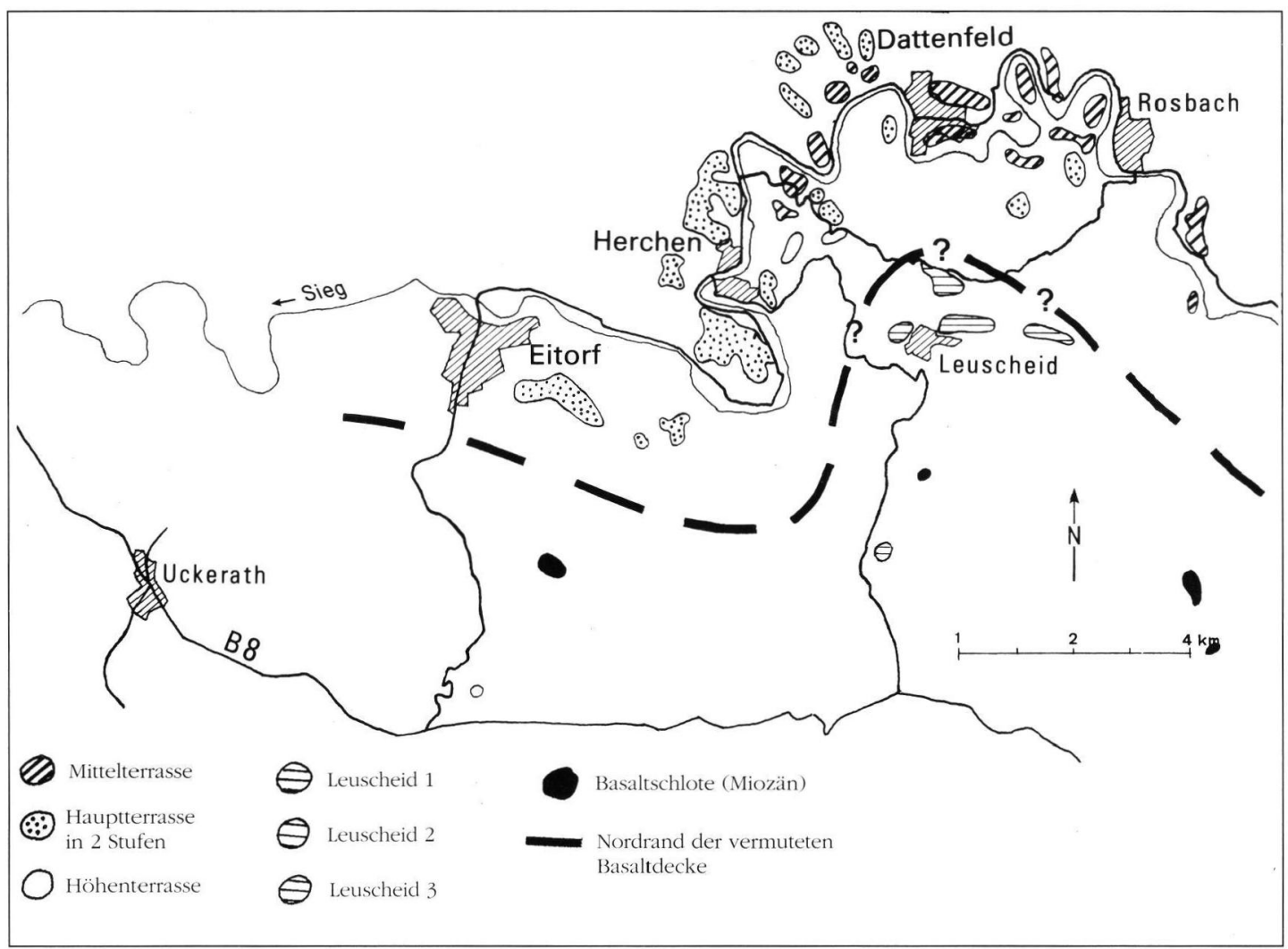

Abb. 3: Der Talmäanderbogen der Sieg bei Dattenfeld und seine Terrassenflächen sowie Verebnungsflächen von Leuscheid Fig. 3: The valley arch of meanders in the Sieg river system near Dattenfeld

Während der Alttertiärzeit hatte sich in dem Gebiet, das durch die spätere Hebung in der Jungtertiärzeit zum Rheinischen Gebirge wurde, eine tiefgründige Zersatzzone auf dem eingerumpften Devon entwickelt. Das damalige Klima des Alttertiärs war „subtropisch" (Paläozän), warm (Eozän) bis warmgemäßigt (Miozän) (ScHWARZBACH 1974: Tab. 23) bei hohen Niederschlägen. Das Alttertiär war global erheblich wärmer als heute, und die Durchschnittstemperatur wird mit im Jahresdurchschnitt über $20^{\circ} \mathrm{C}$ angegeben (SCHWARZBACH 1974: 213). In den Tropen und Subtropen sind rezente Zersatzzonen von über $40 \mathrm{~m}$ Tiefe keine Seltenheiten.

Die polaren Eiskappen existierten damals noch nicht, sie bildeten sich erst nach der Drift des Antarktischen Kontinentes in eine südpolare Lage heraus: die ältesten Glazialsedimente der Antarktis werden in das untere Oligozän gestellt (FLOHN 1985: 176). Der Einfluß des Golfstromes, der nach dem Schließen der mittelamerikanischen Landbrücke seinen Weg in den Pazifik hinein verschlossen fand und zwangsweise nach Norden abgelenkt wurde, um dort das Kondensat seines warmen Wassers als Schnee auf den benachbarten Festländern Kanadas und Skandinaviens sowie Sibiriens abzugeben, ver- ursachte die arktische Vereisung, verzögert um etwa 10 Mio. Jahre (FLOHN 1985: 172: „... daß ein vollvereister antarktischer Kontinent und ein eisfreier arktischer Ozean ... etwa 10 Mio. Jahre lang gleichzeitig nebeneinander existiert haben kann").

In solch einem alttertiärzeitlich tiefgründig zersetztem Gebirgsrumpf fließen natürlich die Niederschläge nicht in "geordneten Bahnen“ $a b$, sondern verwildernd, im besten Falle mäandrierend. Weder bestand damals ein starkes Gefälle zu einem größeren Vorfluter (Strom oder Nordsee), noch gaben harte Gesteine Zwangswege vor. Der Ur-Niederrhein existierte so recht wohl erst ab der heutigen Mosel (die man somit als damaligen Oberlauf des Niederrheines auffassen kann), und seine Zuflüsse mußten sich erst langsam auf den Süd-Nord sich einrichtenden Rhein einstellen. Ein Großteil der Entwässerung des späteren Rheinischen Gebirges war lange Zeit noch nach Süden orientiert. Erst nachdem sich mit der verstärkten Hebung der Rheinischen Masse im Miozän (vgl. auch hierzu Fuchs et al. 1983) das Gefälle verstärkt hatte, konnten die Lockermassen der tiefgründigen Zersatzzonen fortgeräumt und das unverwitterte Gebirge erreicht werden.

Dann aber, als das devonische Gebirge mit seinen Ge- 
steinsgegensätzen - Ton-, Sand- und Schluffsteine, Kalksteine, Quarzite und vulkanische Gesteine - erreicht war, machten diese sich beeinflussend auf den Verlauf wie auch auf die Ablagerungen des rinnenden Wassers bemerkbar. Hatten sich in den weichen Verwitterungszonen bei geringerem Gefälle Flußmäander ausgebildet, wurden diese auch bei nun verstärktem Gefälle beibehalten und als "geführte Mäander" im Festgestein fortgesetzt. Erst eine Änderung der Laufbedingungen (stärkere Wasserführung, kräftigeres Gefälle und zunehmende Geröllfracht) konnte diese Mäander auflösen, aber nur äußerst langsam und recht selten. Eines der schönsten Beispiele für solche geführten Mäander bietet die Sieg zwischen Au und Eitorf (s. Abb. 3) im Talmäanderbogen von Dattenfeld (GRABERT 1975), wo einzelne Schlingen schon abgeschnitten wurden (SCHUMACHER 1931, GRABERT 1981). Aber auch in anderen Flußsystemen sind solche Mäander, wenn auch nicht so exemplarisch schön, vorhanden: an der Wiehl bei Ründeroth (vgl. Abb. 1), an der Bröl bei Herrenstein und an der Wupper. Für den Talmäanderbogen von Dattenfeld müssen daher besondere Ausgangslagen vorhanden gewesen sein; darüber wurde schon diskutiert (GRABERT 1975).

\section{Der Talmäanderbogen von Dattenfeld}

In einem auf wenige Kilometer Laufstrecke beschränkten Abschnitt holt die Sieg aus ihrer angenähert Ostwest verlaufenden Fließrichtung in einem weitgespannten Bogen nach Norden aus (Abb. 3). Diese Laufstrecke ist durch eine Reihe von ausgeprägten Talmäandern, Umlaufbergen und Altwässern ausgezeichnet, von denen einzelne von der heutigen Sieg-Entwässerung nicht mehr durchflossen werden (SCHUMACHER 1931, GRABERT 1981).

Die Hauptterrasse begleitet diesen Mäanderbogen der Sieg in weiter Verbreitung, das gleiche gilt für die Mittel- und Niederterrasse. Einige noch ältere, schottertragende Flächen sind vorhanden; sie werden als Höhenterrassen bezeichnet. Südlich dieses Talmäanderbogens ist um den Ort Leuscheid (ca. $+295 \mathrm{~m} \mathrm{NN}$ ) eine Gruppe von Verebnungsflächen (Leuscheid 1 bis 3) erhalten geblieben. Aus dieser Flächenlandschaft ragen einzelne Bergkuppen als Härtlinge heraus, so der Studchen bei Leuscheid (+ 312,0 m NN) und, als höchster dieser Gegend, „Auf dem Schachten“ (+ 377,2 m NN). Die Flächen Leuscheid 1 bis 3 zeigen außer ihrer ebenen Lage keine weiteren Besonderheiten. Sie tragen keine Sedimente und zeigen auch nur eine geringe, weitgehend rezente Bodenbildung. Gelegentlich sind jedoch an geschützten Stellen Reste fossiler Böden erhalten geblieben.

Die Härtlinge hingegen weisen eine intensive Bleichung der ehemaligen Sandsteine auf und auch ihre besondere Härte ist durch eine Verkieselung, wie sie bei alttertiärzeitlichen Verwitterungsprozessen auftreten, ausgezeichnet.

Die Anlage des Talmäanderbogens bei Dattenfeld ist älter als die Ausbildung der pleistozänen Terrassen der Sieg. Warum dieser Fluß diesen nach Norden ausholenden Bogen gerade hier beschreibt, ist aus heutiger Sicht nicht verständlich; Gesteinsgegensätze, die dieses bewirkt haben könnten, sind im devonischen Grundgebirge nicht vorhanden. Es muß daher die Sieg in früheren Zeiten hier einem Hindernis ausgewichen sein, das sie zu diesem Bogen

Tab. 1: Die Terrassen der Sieg im Talmäanderbogen von Dattenfeld und die Hochflächen von Leuscheid, sowie die Verebnungsgruppen am Hohen Westerwald

Tab. 1: The terraces of the Sieg meandring valley arch near Dattenfeld and the peneplaination near Leuscheid, also these near the

Hohe Westerwald

\section{Holozän}

Holozän

Holozän

Würm-Glazial

Riß-/Mindel-Glazial

Donau-/Biber-Glazial

Alt-Pleistozän

Pliozän

(Jung-)Miozän

(Alt-)Miozän

Oligozän

Oligozän
Siefen-Bildung

Talaue der Sieg

"Inselterrasse“

Niederterrasse

Mittelterrasse

Hauptterrasse

Höhenterrasse

"Leuscheid 1"

"Leuscheid 2"

"Leuscheid 3“

gebleichte Härtlinge

Prä-Basalt-Fläche

$\begin{array}{ll}+95 \mathrm{~m} & \text { Siegtal bei Dattenfeld } \\ +100 \mathrm{~m} & \text { Siegtal bei Dattenfeld } \\ +105 \mathrm{~m} & \text { Brücke bei Dattenfeld } \\ +120 \mathrm{~m} & \text { Wilberhofen } \\ +180 \mathrm{~m} & \text { Röcklingen bei Herchen } \\ +205 \mathrm{~m} & \text { Röcklingen bei Herchen } \\ +230 \mathrm{~m} & \text { Leidhecke, Türmchenseiche } \\ +280 \mathrm{~m} & \text { Sangerhof }\end{array}$

$+310 \mathrm{~m}$

$+360-390 \mathrm{~m}$

$+390 \mathrm{~m}$ mehr
Obersaal, Spurkenbach

A. d. Schachten, Giebelwald

Beulskopf
IIb: $+390-480 \mathrm{~m}$

IIa: $+420-525 \mathrm{~m}$

I: $460-480 \mathrm{~m}$ 
zwang. Dieses Hindernis ist heute nicht mehr vorhanden, es war, so wird vermutet, eine mehr oder weniger zusammenhängende Decke aus miozänen Basalten.

Die Basaltdecken des Hohen Westerwaldes im Süden und das devonische Grundgebirge des Bergischen Landes im Norden werden durch eine ungefähr Ost-West verlaufende Linie begrenzt; dieser Linie folgte die frühe Sieg-Drainage, als sie sich nach dem Miozän auf den im Westen fließenden Rhein ausrichtete.

Nun beschreibt dieser Fluß bei Dattenfeld den so unmotivierten Bogen nach Norden. Dieser wird jedoch erklärbar, wenn man die Vorkommen einzelner Basaltschlote südlich dieser Linie im Gebiet von Leuscheid betrachtet (Beulskopf, Pt. 316,8 nördlich von Busenhausen, Pt. 360 östlich von Kuchhausen). Diese Schlote stellen die Förderkanäle dar, die im Miozän an der damaligen Landoberfläche eine größere, zusammenhängende Basaltdecke produzierten. Diese Decke wurde in nachfolgender Zeit ein morphologisches Hindernis in der sich zum Rhein hin ausrichtenden Drainage. Der Fluß mußte nach Norden - ausweichen und in einem Bogen dieses Hindernis umfahren. Daß in diesem Bogen so viele Mäanderschlingen angelegt wurden, ist auf das - vermutlich auch im Miozän begonnene oder verstärkte - Einsinken der Bergischen Muldenzone zurückzuführen. Dadurch entstand ein Gebiet mit geringem Gefälle. Die einmal in der tiefgründig verwitterten flachen Landschaft angelegten Mäander wurden bei Erreichen des Grundgebirges zwangsläufig eingetieft. So entstanden die geführten Gebirgsmäander, an die sich im Pleistozän, entsprechend dem klimatischen Wechsel, schottertragende Terrassenflächen (von der Höhen- bis zur Niederterrasse) anlehnten.

So ungewöhnlich solche Mäanderstrecken im heutigen Mittelgebirge sind, so weisen sie doch einige Besonderheiten auf: sie liegen alle in postorogenen, heute als „saxonisch“ gedeuteten Senkungszonen. Der Talmäanderbogen der Sieg bei Dattenfeld liegt in der Bergischen Muldenzone (GraBerT 1983), und auch die Mäanderstrecke der Mosel zwischen Trier und Cochem, besonders die in der Wittlicher Senke (NEGENDANK 1983: Abb. 5), liegen in einer - weit größeren - Senkungszone, in der ebenfalls postorogenen Eifeler Nordsüdzone, in der sich Reste der einst ausgedehnteren Buntsandstein-Bedeckung erhalten haben.

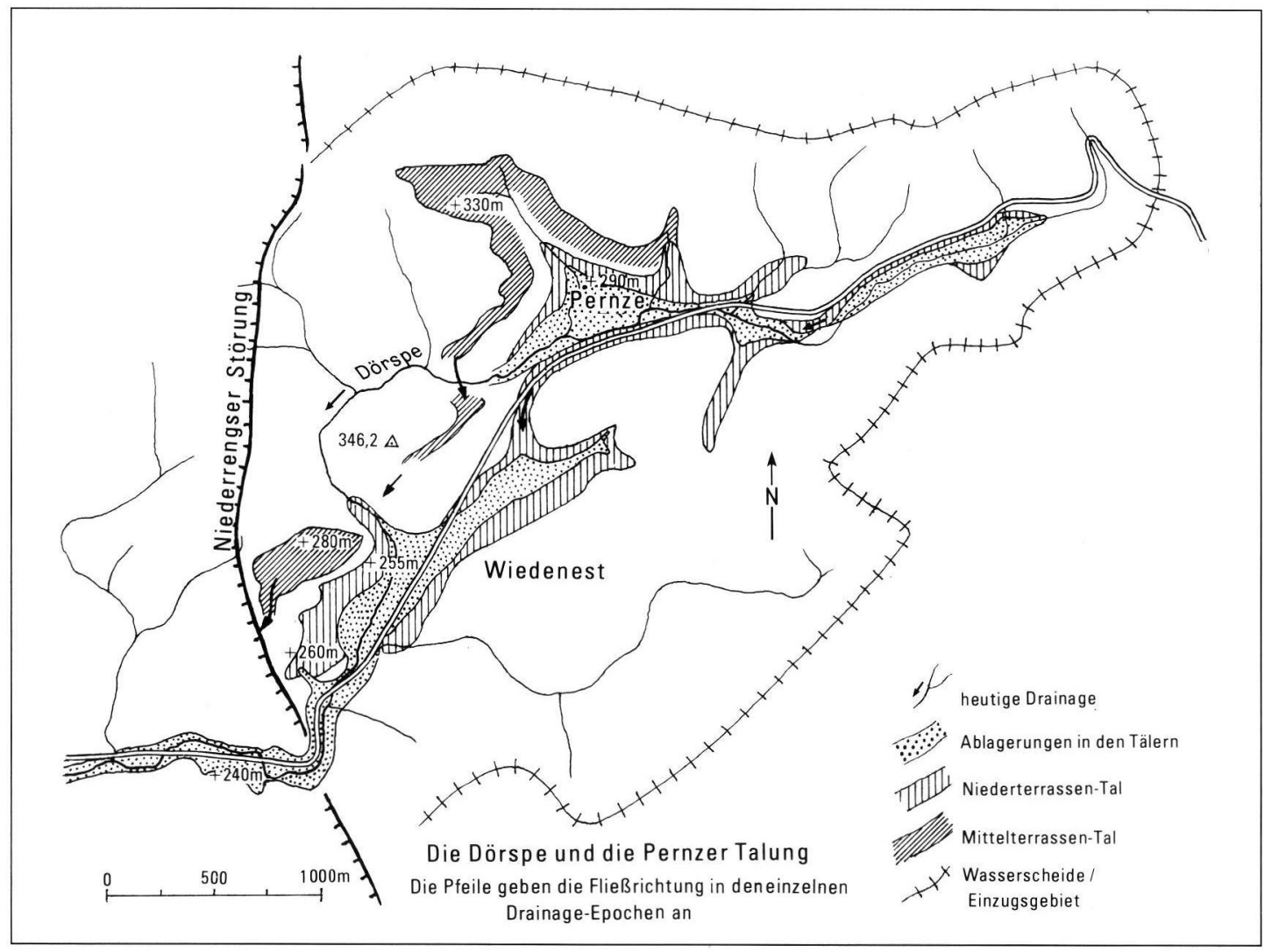

Abb. 4: Die Pernzer Talung am Oberlauf der Agger bei Bergneustadt

Fig. 4: The Pernze head valley in the Agger-Dörspe drainage system 
Nach dem eben Gesagten müßten sich mit Erreichen des devonischen Gebirges die Täler auf ihren vorgezeichneten Bahnen kontinuierlich eintiefen, nur abhängig von der Hebung der Rheinischen Masse und - etwas auch - vom Gesteinsgegensatz sowie von klimatischen Einflüssen. Die schon erwähnten Laufveränderungen der Sieg im Talmäanderbogen von Dattenfeld lassen aber auch eine Diskussion einer tektonischen Beeinflussung zu. Diese könnte sich durch eine Zerblockung der sich hebenden Rheinischen Masse anzeigen und zwar dergestalt, daß diese Hebung nicht gleichmäßig, sondern sich wölbend bemerkbar macht, so daß der Gebirgskörper gedehnt und von zerrenden Störungen durchsetzt wird; diese bieten sich dann als Abschiebungen dar. Erkennbar wären solche Störungen nur an Verstellungen von Terrassenflächen und/oder an einer sprunghaften Veränderung in der Gefällekurve der Fließgewässer. Ein solches Beispiel ist am Oberlauf der Agger gegeben: an der Pernzer Talung.

\section{Die Pernzer Talung}

Die Pernzer Talung (Abb. 4) bildet den eigentlichen Oberlauf der Agger. Der am Schnüffel bei Meinerzhagen entspringende Bach hat zwar eine größere Wasserführung als die von der Wasserscheide bei Wegeringhausen in Ost-West-Richtung fließende und dann unterhalb Bergneustadt in die Agger einmündende Dörspe, doch sollte dieser als Pernzer Talung bezeichnete Oberlauf des Agger/Dörspe-Systems wegen seiner Ost-West-Gesamtfließrichtung als sinngemäßer Oberlauf des Agger-Systems angesehen werden.

Auf der gut $5 \mathrm{~km}$ langen Fließstrecke der Dörspe von Pernze bis zu ihrer Einmündung in die Agger bei Derschlag (unterhalb Bergneustadt) besteht ein Gefälle von rund $70 \mathrm{~m}$, das sind $14 \mathrm{~m}$ auf $1 \mathrm{~km}$ Flußstrecke. In der Pernzer Talung hingegen ist das Gefälle geringer und beträgt nur $5 \mathrm{~m}$ auf $1 \mathrm{~km}$, obwohl sonst in den meisten Oberläufen eines Gewässersystemes die Gefällekurve stärker geneigt ist.

Die Pernzer Talung wird vom eigentlichen Aggertal durch ein sehr enges Talstück, durch die Engstelle an der Kirche von Wiedenest, getrennt. An dieser Stelle wird die Dörspe nur von einem geringmächtigen und schmalen Schotterkörper begleitet, der hier im Niveau der Talaue liegt. Andere, also ältere Terrassenflächen, sind in dieser Engstelle nicht ausgebildet, obwohl sie oberhalb, also in der Pernzer Talung, vorhanden sind. Dennoch: die - ältere - Mittelterrasse ist in der Nachbarschaft der besagten Engstelle wohl vorhanden und zeigt an, daß die Dörspe schon damals das Gebiet des Oberlaufes entwässerte. Gleiches gilt auch für die Niederterrassenfläche. Nach der Ausbildung der Niederterrassenfläche mit dem Absatz ihrer entsprechenden Schotter hat die
Dörspe ihren Lauf verlegt. Ein Grund ist keineswegs eindeutig zu erkennen, zumal dies vom Gestein her nicht anzunehmen ist. So bleiben tektonische Bewegungen für eine Laufveränderung im Gewässernetz der Dörspe diskussionsfähig.

Die geologische Karte zeigt nämlich an der Engstelle von Wiedenest eine große Nord-Süd-Störung, die Niederrengser Störung (Abb. 4). Diese wird als ein relativ junges, „saxonisch“ angelegtes Element angesehen, das im Zuge der Bildung der Bergischen Muldenzone (GraBERT 1983) angelegt wurde und diese Zone langaushaltend begleitet. An ihr sind an einer antithetischen Störung im Westen die devonischen Schichten um mehrere hundert Meter abgesunken. Diese Niederrengser Störung ,trennt" die Pernzer Talung vom eigentlichen Aggertal, nicht nur dadurch, daß das Gefälle in der Pernzer Talung geringer als im oberen Aggertal bei Bergneustadt ist (was ungewöhnlich ist), sondern dadurch, daß diese Störung in geologisch junger Zeit wieder aufgelebt ist und diese Trennung verursacht hat. Geschehen ist diese Trennung nämlich nach der Ausbildung der Niederterrasse, die, zusammen mit der Mittelterrasse, durch diesen Vorgang extreme Laufveränderungen erfahren hatte, die bis in jüngste geologische Zeiten anhielten. Das Mittelterrassental der Dörspe läßt sich anhand der erhaltenen Verebnungsflächen rekonstruieren (Abb. 4). Es setzt nördlich des Ortes Pernze ein, endet also nicht an der heutigen Wasserscheide bei Wegeringhausen, wie etwa das Tal der Niederterrasse.

Das Mittelterrassental zieht dann südlich der Höhe +346,2 nach Westen auf die Niederrengser Störung zu. Ungefähr bei der Höhe $+310,0$ westlich von Wiedenest endet die Niederterrassenfläche der Dörsper Talung ganz plötzlich an der hier durchziehenden Niederrengser Störung; die Mittelterrasse hat hier eine Seehöhe von $+280 \mathrm{~m}$ NN. Westlich von ihr, nun aber im Aggertal, setzt die nächste Mittelterrassenfläche bei $+265 \mathrm{~m}$ NN ein. Dieser Vertikalverwurf von etwas weniger als $20 \mathrm{~m}$ auf relativ kurzer Entfernung wird durch eine tektonische Bewegung im Zuge der noch immer nicht abgeklungenen Herausbildung der Bergischen Muldenzone erklärt.

Auch die Niederterrasse der Dörspe zeigt noch eine ähnliche Entwicklung wie die Mittelterrasse auf. Die Niederterrasse setzt fast an der Wasserscheide bei Wegeringhausen ein und übernimmt somit nicht mehr die Ursprungsrichtung der Mittelterrasse nördlich von Pernze. Schon deutlich nach Süden abgesetzt, jedoch parallel zum Mittelterrassental, entwickelt die Dörspe ihr Niederterrassen-Bett und endet ebenfalls abrupt an der Niederrengser Störung bei einer Seehöhe von $+260 \mathrm{~m} \mathrm{NN}$.

Erst bei Bergneustadt ist die Niederterrasse dann wieder nachzuweisen, fehlt also wie die Mittelterrasse auf der ganzen ca. 2,5 km langen Engstelle von 


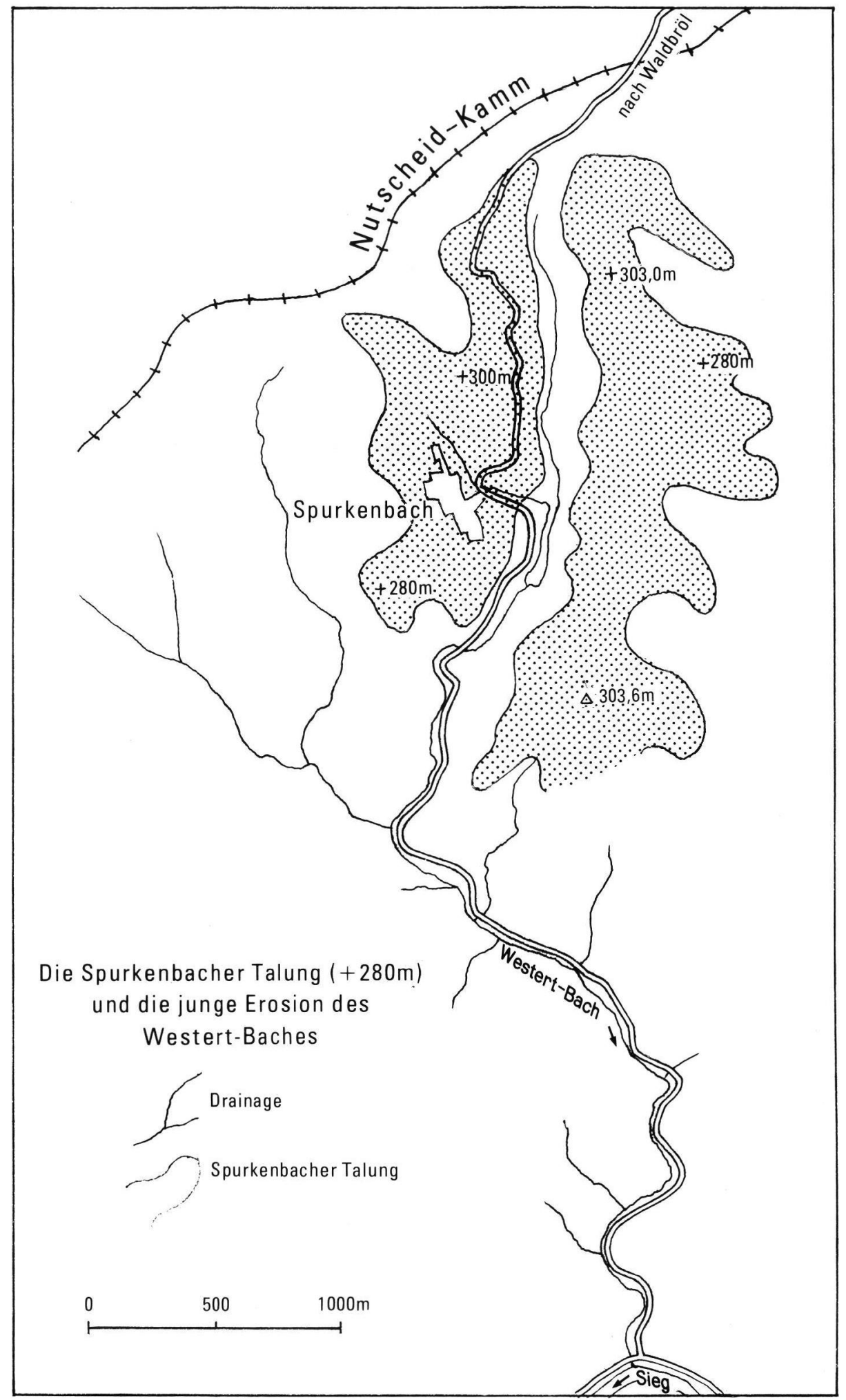

Abb. 5: Die Spurkenbacher Talung des Westert-Baches (Sieg-Nebenbach)

Fig. 5: The erosion surface Spurkenbach and its capture by the Westert brook 
Wiedenest. Erst die heutige Talaue bildet einen durchziehenden und geschlossenen Schotterkörper vom Oberlauf oberhalb Pernze bis zur Einmündung in die Agger bei Derschlag.

Diese junge Talbildung hat die NiederterrassenFlächen kurz vor Beginn der Engstelle bei Wiedenest in rückschreitender Erosion angeschnitten (Abb. 4).

Noch in geologisch junger Zeit hat die Dörspe in der Pernzer Talung ihren Lauf aus den älteren, durch Talboden und Terrassen gekennzeichneten Talverlauf verlassen und neue Wege durch bisher nicht berührte Gebirgsteile gesucht. Dort, wo in Abb. 4 der kleine Pfeil - er steht für die Fließrichtung der Dörspe und ist oberhalb des Nord-Pfeiles vermerkt eingezeichnet ist, verläßt der Fluß seinen Talboden und hat sich südlich davon im devonischen Gebirge eine um mehrere Meter tiefe Schlucht eingegraben. Besonders auffällig ist das Herauslaufen der rezenten Dörspe aus den älteren Terrassenflächen dann östlich der Niederrengser Störung. Westlich von Pernze verläßt sie nämlich die junge Talaue, kreuzt sogar ihre alte Mittelterrassen-Fläche und umfließt in einem weiten Bogen die heutige Höhe $+346,2 \mathrm{~m} \mathrm{NN}$, die in ihrer Gesteinszusammensetzung jedoch keinen Grund für ein derartiges Verhalten besitzt. Folgt man den Untersuchungen von Nicke (1989), der solche tief eingeschnittenen und steilen Gebirgshänge betrachtet hat, dann wären diese jüngsten Abweichungen im Flußregime der Agger/Dörspe holozän bis rezent und somit die jüngste Reliefgeneration des Bergischen Landes. Sie entstanden, als nach dem extremen pleistozänen Klima hier im Mittelgebirge noch kaum wieder eine Vegetation vorhanden war. Damit ist zwar das Alter solcher Siefenbildung gegeben, nicht aber deren Ursache. Ähnliches gilt auch für die Spurkenbacher Talung.

\section{Die Spurkenbacher Talung}

Die Spurkenbacher Talung (zur Lage s. Abb. 1) liegt südlich des Nutscheidkammes und besteht aus einer ca. $3 \mathrm{~km}$ langen und $1,5 \mathrm{~km}$ breiten Verebnungsfläche mit einer Meereshöhe um $+300 \mathrm{~m}$ NN (Abb. 5). Außer einer wesentlich jüngeren Bedeckung von teilweise recht mächtigem Lößlehm trägt diese Talung keine weiteren Sedimente. Jüngere Verebnungsflächen oder gar schottertragende Terrassenflächen sind an ihren Rändern nicht vorhanden. Heute wird sie vom Westertbach zur Sieg hin entwässert. Lange Zeit muß diese Talung isoliert gewesen sein. Erst in relativ junger Zeit, wobei offen bleibt, wann das geschehen ist, hat sich der Westertbach in rückschreitender Erosion aus dem Siegtal zum Nutscheidkamm durchgearbeitet und dann die Spurkenbacher Talung angeschnitten.

Zieht man das gut mit Verebnungs- und Terrassen- flächen ausgestattete Gebiet an der Sieg und um den Ort Leuscheid zum Vergleich heran, läßt sich die Spurkenbacher Talung am ehesten mit der Verebnungsfläche „Leuscheid 3“ (+310 m NN) oder ,Leuscheid 2“ (+280 m NN) parallelisieren (Tab. 1 und Abb. 3).

$\mathrm{Zu}$ fragen ist nach dem Alter der Anzapfung der Spurkenbacher Talung durch den Westertbach. Da diese Talung nicht von pleistozänen Terrassenflächen begleitet wird, muß die Anzapfung jünger sein. Es wird daher angenommen, daß diese zur Niederterrassenzeit erfolgt ist, wenn man nicht sogar, in Anlehnung an Nicke (1989) ein holozänes Alter, also nach der Eiszeit, annehmen will. Ob auch hier tektonische Ursachen der so späten Anzapfung durch den Westertbach möglich sind, wie man es in der Pernzer Talung anzunehmen geneigt ist, bleibt offen. Es wäre auch zu diskutieren, ob die restlose Erosion der postulierten Basaltdecke von Leuscheid eine so späte Anzapfung bewirkt hat.

\section{Zusammenfassung}

Der Anfang und der Umbau des rechtsrheinischen Gewässernetzes zwischen Wupper und Sieg weist zwei Großepochen auf, dic durch klimatische wie auch tektonische Prozesse ihre Ausgestaltung erhalten haben.

Im Alttertiär bildeten sich durch die feuchtwarme (,tropoide“) Verwitterung tiefreichende Zersatzzonen aus, auf denen die abfließenden Niederschläge verwildernd und mäandrierend abflossen. Vermutlich wurde ein großer Teil des damals noch in niederer Seehöhe als heute liegenden späteren Rheinischen Gebirges nach Süden (in das Mainzer Becken?) entwässert.

Im Miozän setzte, initiiert vermutlich durch die Alpen-Orogenese, die Hebung der Rheinischen Masse ein. Dieser Hebungsprozeß lief als eine Hochwölbung ab, begleitet von zerrenden Störungen und einsinkenden Depressionen. Die ersten Trogflächen bildeten sich heraus, die vom ablaufenden Wasser benutzt wurden; es entstanden die Trogtäler. Begleitet wurde die Hochwölbung vom miozänen BasaltVulkanismus, der die weitere morphogenetische Entwicklung wesentlich beeinflußte. Der „Morphogenetische Umbruch" steht am Anfang dieser Entwicklung.

Die ältesten fluviatilen Sedimente auf dem devonischen Grundgebirge des Bergischen Landes und des südlich anschließenden Niederen Westerwaldes sind die hellen, weitgehend aus weißen Quarzen (mit untergeordneten Lyditen und Hornsteinen) sich zusammensetzenden Schottern; sie werden als „Vallendarer Schotter" bezeichnet und in das Pliozän gestellt. Sie treten nur am Gebirgsrande auf und zeigen keinerlei Beziehungen zu irgendwelchen rezenten Flußsystemen. 
Die Quarzkiese sind ein Aufbereitungsprodukt des tertiärzeitlichen „tropoid" verwitterten Gebirges. Reste dieses so veränderten Gebirges sind noch vereinzelt erhalten geblieben, die heute als Härtlinge aus den tertiärzeitlichen Altflächen herausragen. Die Höhe „Auf dem Schachten“ mit $+377,2$ m NN in der Hochflächengruppe von Leuscheid (TK 25 Weyerbusch 5211) kann hier genannt werden.

Das Pleistozän, vermutlich schon im oberen Pliozän einsetzend, steuert nun mit seinem kühleren Klima und den stärkeren Wassermengen die weitere Entwicklung des Gewässernetzes.

Gleichzeitig hebt sich die Rheinische Masse verstärkt und wird zu einem stark zertalten Gebirge umgestaltet. Alle größeren Flüsse im Rechtsrheinischen bilden nun schottertragende Terrassenflächen aus: die Höhenterrasse (nur an den größeren Flüssen Sieg und Ruhr), die Haupt-, Mittel- und Niederterrasse, jeweils eng an den Flußverlauf gebunden. Diese Flächen korrespondieren mit solchen des Rheintales, womit kenntlich wird, daß sich das rechtsrheinische Gewässernetz auf den Vorfluter Rhein eingestellt hatte.

Es ist logisch, daß die großflächige Hebung der Rheinischen Masse nicht kontinuierlich ablaufen konnte und damit auch nicht wirkungslos auf die Gestaltung des Gewässernetzes blieb. Eine Hochwölbung geht mit einer Dehnung des Gebirgskörpers einher, und dieser läßt sich nicht unbegrenzt dehnen. Es reißen Spalten und Brüche auf, die zu einer Zerblockung des Gebirges führen. Dabei wurden ältere Störungen auch einmal reaktiviert. Solche Ereignisse wirken intensiv auf das Gewässernetz ein, da genügen oft nur wenige Meter Änderung in der Flußgefällekurve. Ein eindrucksvolles Beispiel zeigt der Oberlauf der Agger, die als Dörspe die Pernzer Talung entwässert. Hier hat nach der Niederterrassenzeit, anscheinend sogar noch in früher holozäner Zeit, eine wechselhafte Umgestaltung dieses Flußabschnittes stattgefunden. Ein besonders starkes Einschneiden mit steilen Talflanken hat in der vegetationslosen Zeit an der Wende vom Pleistozän zum Holozän im Zusammenspiel mit einem höheren Wassserangebot stattgefunden. Hier können kleinste tektonische Bewegungen von nur wenigen Metern den Abfluß beeinträchtigen.

\section{Schriftenverzeichnis}

Birkenhauer, J. (1970): Der Klimagang im Rheinischen Schiefergebirge und in seinem näheren und weiteren
Umland zwischen dem Mitteltertiär und dem Beginn des Pleistozäns, - Erdkd., 24: 268 - 284; Bonn

FEY, M. (1974). Geomorphologische Untersuchungen im Bergischen Land (Rheinisches Schiefergebirge). - Düsseldorf. geogr. Schr., 1: 181 S.; Düsseldorf

Flohn, H. (1985): Das Problem der Klimaveränderungen in Vergangenheit und Zukunft. - Erträge der Forschung, 220: 228 S.; (Wiss. Buchges.), Darmstadt

FuCHs, K. et al. (1983): Plateau Uplift - The Rhenish Shield. - 411 S., (Springer-Verlag), Heidelberg

Grabert, H. (1975): Der Talmäanderbogen der Sieg zwischen Au und Eitorf (Rheinisches Schiefergebirge). Decheniana, 127: 145-156; Bonn

- (1981): Bau und Entstehung des Umlaufberges von Dreisel/Sieg im Talmäanderbogen von Dattenfeld (rechtsrheinisches Schiefergebirge). - Sonderveröff. Geol. Inst. Köln, 41: 69 - 82; Köln

- (1983): Die Bergische Muldenzone des rechtsrheinischen Schiefergebirges. - Decheniana, 136: 85-94; Bonn

GramsCH, H.-J. (1978): Die Entstehung des Siegtals im jüngsten Tertiär und im Quartär. - Bochum geogr. Arb., 196 S. (Schöningk), Paderborn

Hoos, L. (1936): Die Oberflächenformen zwischen Agger und Sieg. - Decheniana 93: 113-176; Bonn

Knuth, H. (1922): Die Terrassen der Sieg von Siegen bis zur Mündung. - Centr. bl. Min. M.ber., 1922: 336-344; Stuttgart

Lippolt, H. J. (1983): Distribution of volcanic activity in space and time. - In: Fuchs et al., Plateau Uplift, 112120, (Springer-Verlag), Heidelberg

Negendank, J. (1983): Cenozoic deposits of the Eifel-Hunsrück area along the Mosel river and their tectonic implications. - in: FucHs et al., Plateau Uplift 78-88 (Springer-Verlag), Heidelberg

NickE, H. (1983): Reliefgenese des südlichen Bergischen Landes zwischen Wupper und Sieg. - Köln geogr. Arb., 43: 278 S., Köln

- (1989): Siefen - Geomorphologische Untersuchungen an einer Sonderform der Talanfänge im Bergischen Land. - Decheniana, 142: 147-156, Bonn

PhILIPpson, A. (1899): Entwicklungsgeschichte des rheinischen Schiefergebirges. - Verh. Nat. hist. Ver. preuß. Rheinld. u. Westf., Sitz.-ber., 56: 48-50; Bonn

QuiTzOW, H. W. \& WAGNeR, W. \& WiTTMANN, Q. (1962): Die Entstehung des Rheintales vom Austritt des Flusses aus dem Bodensee bis zur Mündung. - Beitr. Rheinkd., 14: 9-47; Koblenz

SCHRÖDER, E. (1965): Zur Talgeschichte der unteren Sieg. Decheniana, 118: 41-45; Bonn

- (1969): Alter und Entstehung der rechtsrheinischen Trogflächen zwischen Agger und Sieg. - Decheniana, 122: 21-29; Bonn

Schumacher, A. (1931): Der tote Siegarm bei Schladern. Nachr. Bl. oberberg. Arb. - Gem. naturwiss. Heimatforsch., 2: 31-34; Gummersbach

SchwarzBACH, M. (1974): Das Klima der Vorzeit. - $380 \mathrm{~S}$. (Enke-Verlag); Stuttgart

Manuskript eingegangen am 25.04.1994 